\title{
The Venus Transit, the Mayan Calendar and Astronomy Education in Guanajuato, Mexico
}

\author{
H. Bravo-Alfaro, C. A. Caretta, E. M. S. Brito, P. Campos and \\ F. Macias \\ Universidad de Guanajuato, Mexico, \\ email: hector@astro.ugto.mx
}

In this work we present two aspects of the Astronomy education activities carried out in 2012 by a multidisciplinary group at Universidad de Guanajuato, including specialists in Astronomy, Social Sciences and Environmental Engineering. The first program linked the Venus Transit, occurred in June 2012, with a national campaign of vulgarization of both modern and ancient (Mayan) Astronomy. Professional astronomers all around the country took advantage of the recent myth linked to the end of a large Mayan calendar cycle (13 baktuns, or some 5125 years) happening, after certain authors, in December 2012. In Guanajuato, the Astronomy Department organized live observations of the Venus Transit at two different locations, and complemented with conferences about astronomical events and the fake predictions of disasters linked to the "end" of the Mayan calendar. This program was very successful not only in Guanajuato but throughout the country, with several thousands of people attending live observations, conferences, expositions, etc.

The second program described in this work started back in 2009, in collaboration with researchers of the Culture and Society Studies Dept., who are specialized in rural development. After the great success of the first national star gazing (Noche de las Estrellas 2009), being part of the International Year of Astronomy in Mexico, several communities across the central region of Mexico (in the state of Guanajuato) requested our support in order to organize their own star gazing. Nowadays, we have visited more than a dozen towns, with several hundred visitors at each run (mainly children), most of them watching the sky through a telescope for the very first time. Now we have the collaboration of people from the Environmental Engineering Group, who are actively working to improve the conditions of water, soil and the forests in general, of those spots that have been included in our program.

More information about both these programs may be seen at the web site, http://www.astro.ugto.mx and at: El sujeto cultural y los studios multidisciplinarios, Macias \& Campos eds. Universidad de Guanajuato. 2012 in press. 